\title{
AUTOMATIC ANALYSIS AND CLASSIFICATION OF THE AIRIX SINGLE SHOT ACCELERATOR DEFAULTS
}

\author{
E. Merle, J. Delvaux, M. Mouillet \\ CEA - PEM F51490 Pontfaverger - France \\ J.C. Ribes, G. Delaunay \\ LAM - Université de Reims Champagne-Ardenne - F51687 REIMS cedex2
}

\section{Abstract}

The AIRIX facility is a high current linear accelerator (2-3.5kA) used for flash X-ray radiography at the CEA of Moronvilliers (France). The general background of this study is the diagnosis and the predictive maintenance of the AIRIX components. We are interested to the performances of the HV generators, which furnish the energy to accelerate the beam.

The single shot functioning imposes to obtain the best performances at a given time. So we study and develop a prototype of monitoring tool using neural network and pattern recognition.

Statistical models are used to define an error vector, representative of the state of the generators, which must be classified. To reduce the redundancy of this information and the computation time, we study two algorithms, the principal component analysis and the curvilinear components analysis. A classifier has been defined, with a three layer Radial Basis Function (RBF) neural network. We initialize the network by applying an unsupervised fuzzy technique to a training base. The configuration of the whole net is realized by a supervised training. Continuous learning must be enable to take in account new states, and to monitor the experiments to predict future failures. We will present the recent results obtained on the installation.

\section{INTRODUCTION}

The AIRIX accelerator [1] creates and accelerates an intense electron beam (3.5kA, $60 \mathrm{~ns})$ for flash radiography. The single shot functioning needs to be optimal at the desired time. We try to define a supervision method for fault diagnosis for the AIRIX facility. The objective is to guaranty an optimal functioning and to search precursors for the known failures in developing predictive maintenance. We are interested in diagnosis using pattern recognition with neural network, and particularly RBF nets. RBF neural nets are employed for supervised classification. We propose an original approach to construct RBF nets for unsupervised classification. This classifier is improved by a procedure allowing to update the net with new states in a continuous learning.

In a first paragraph, we will explain the experimental context of the study and the wished results. After some recalls on the construction strategy, we will review the different results we obtained on the first years experiments data.

\section{EXPERIMENTAL CONTEXT}

The AIRIX induction accelerator is composed by two main sets. The accelerator part and the electrons source, the injector. At each experiment there are about thousand parameters to observe.

The major objective of our study is to optimize the functioning of the machine and to develop predictive maintenance by improving the diagnosis.

We use signal processing and pattern recognition to select and to recognize the different functioning states of the accelerator.

In a first approach, this type of analysis is a way to highlight signal variations to search imperfect functioning modes on the installation

Secondly we have defined a strategy to construct a classifier with unsupervised data, able to recognize failures and to take in account new states in a continuous learning [2]. We recall the general principle of this procedure, while insisting on the definition of the error vector to classify and on a data mining algorithm, the curvilinear components analysis (CCA). This algorithm gives us best performances than the principal components analysis used for linear dependencies search.

This classifier allows a quick view of the state of functioning of the active elements of the machine and is now used on the accelerator.

\section{SIGNAL PROCESSING AND DATA MINING}

The aim of those algorithms is to reduce the dimension of the data and to select the most pertinent parameters. We have defined an average model of the output signal of the generator, associated with a $2 \sigma$ confidence interval. On the one hand, this model allows to define an error vector to classify while preserving the information of the signal. On the other hand the defined error is more robust than using parameters and permits to introduce a tolerance on the physical variation of the functioning.

The error vector is defined by the following formulas.

$\left\{\begin{array}{l}\text { if } s(t)>\bar{s}(t)+2 * \sigma(t) \Rightarrow \operatorname{err}(\mathrm{t})=s(t)-(\bar{s}(t)+2 * \sigma(t)) \\ \text { if } s(t)<\bar{s}(t)-2 * \sigma(t) \Rightarrow \operatorname{err}(\mathrm{t})=s(t)-(\bar{s}(t)-2 * \sigma(t)) \\ \operatorname{err}(\mathrm{t})=0 \text { elseif }\end{array}\right.$ 
$\bar{s}(t)$ and $\sigma(t)$ are respectively the mean and the standard deviation of the signals for the $\mathrm{t}^{\text {th }}$ sample.

The next figure represents the error obtained for a good functioning signal.

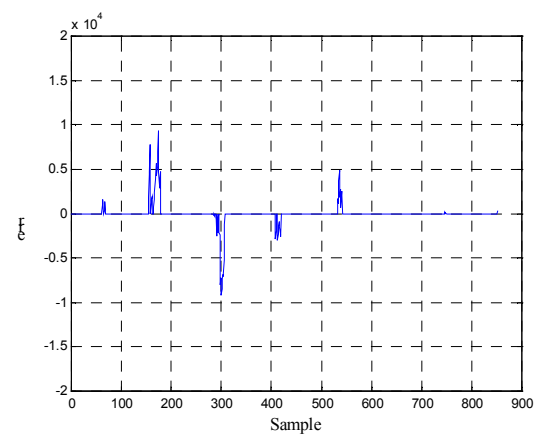

Figure 1 : error vector

The major drawback of this approach is the calculus time, because we conserve a vector of size 850 .

We have previously used the PCA to reduce the dimension of the problem. For the generators analyze, the CCA has given the best results.

The CCA algorithm has been proposed by Demartines [3]. It is based on a non-linear projection of the data on a lower dimension space, that is to say to unfold a space of dimension $\mathrm{n}$ on a space of lower dimension $\mathrm{p}$.

This property is illustrated on the next scheme.

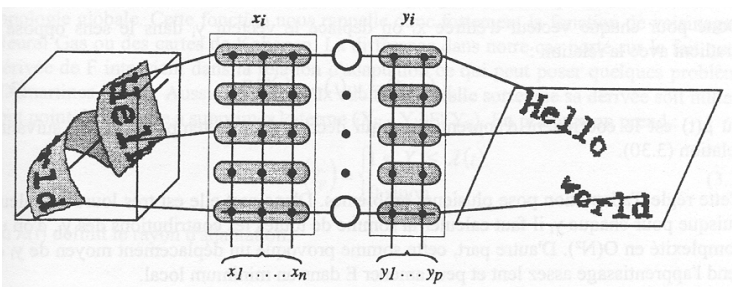

Figure 2 : property of the CCA

Mathematically, it is expressed by the following criterion to minimize.

$E=\frac{1}{2} \sum_{i} \sum_{j \neq i}\left(d\left(x_{i}, x_{j}\right)-d\left(y_{i}, y_{j}\right)\right)^{2} F\left(d\left(y_{i}, y_{j}\right)\right)$

This algorithm can be put under the form of a network with two layers, where the first one makes a vectorial quantization of the $\mathrm{n}$ dimensional input space and the second one realized a non-linear projection on a $\mathrm{p}$ dimensional space.

The self-organization is realized by forcing the distances in the output space to match the distances in the input space, according to the precedent criterion. In other words, we try to obtain the conservation of the distances during the non-linear projection. This principle is iterated to the whole couples of vectors of the space.

With this algorithm, we have obtained the decision space represented here under.

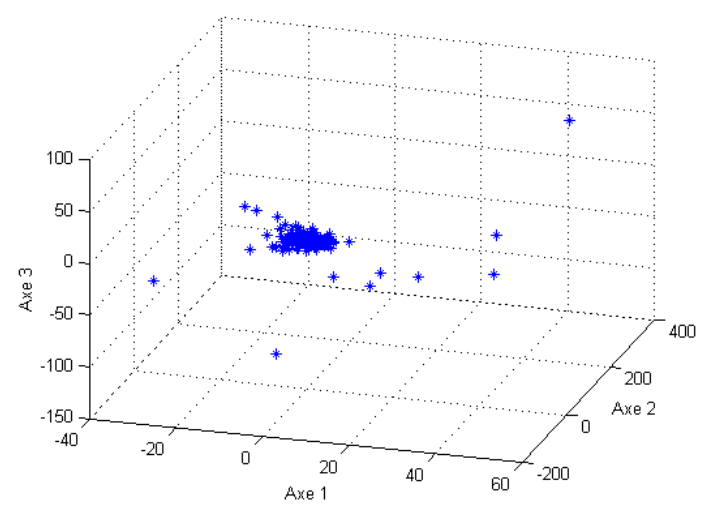

Figure 3 : Space obtained with the CCA

\section{RBF NET CLASSIFIER}

Previous results [4] permit us to test our construction procedure. Those results have been obtained with data of small dimension (only 3 components). The data issued of the generator of the AIRIX installation have 20 dimensions. The precedent figure is obtained by the data projection on the three first components.

The principle of our procedure is resume by the next scheme.

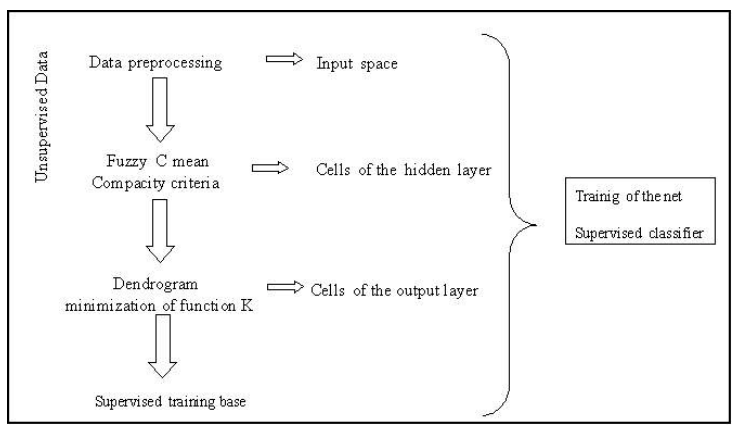

Figure 4 : proposed construction procedure

The first step of this procedure is to extract the information necessary to characterize a situation or a state. This information is reduced by the precedent algorithm and analyzed in an unsupervised manner to detect the different classes present in the space. The last step is to initialize the classifier with the defined training base.

We choose to use RBF neural network in the one hand for their good properties of localization (with the RBF kernel) and of generalization. On the other hand this type of network accepts the reject options which are necessary to enable the continuous learning.

\section{RESULTS}

We illustrate this paper by the most recent results we obtained on the injector and on the generators of the AIRIX linac. 


\subsection{Injector}

The next picture represents the decision space defined with the prime power data. We note the transient class, which comes before the failure.

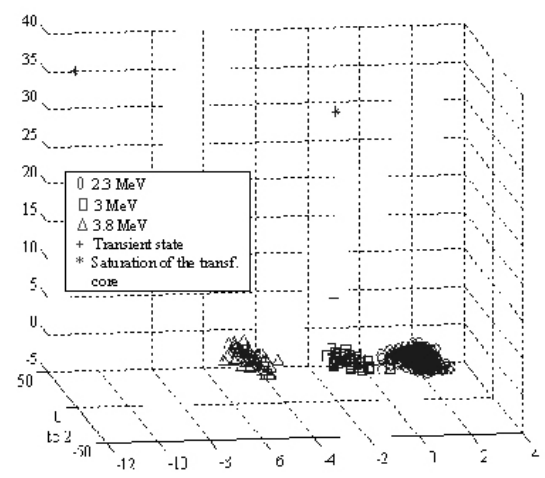

Figure 5 : Prime power decision space

This results is very interesting, because we show that our approach can be used to predict failures and enable an optimization of the maintenance plan.

\subsection{HV generators}

The dimension of the defined space is much high that for the injector. We reduce the size of the problem to 20 parameters with the CCA. We present the results on the first three components.

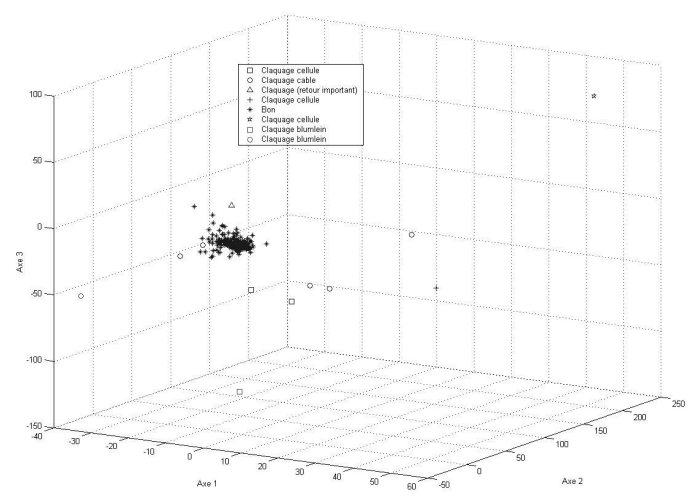

Figure 6 : Diagnosis of the generators

The discrimination of the classes is not so easy than the injector states. This is linked with the important possible variations of the signal. We notice that a same phenomenon is represented by various classes. This remark is particularly true for the electrical breakdown. With the type of electrical breakdown the measured signature on the generator signal is not exactly the same. This representation allows us to show the different problem which can appear on the cells.

\section{CONCLUSION}

In this paper we have presented the most recent results obtained on the diagnosis of the AIRIX installation. The precedent results have been applied to the generator analysis and have given good performances. The automatic classification is an help for the functioning optimization and can permit to improve the maintenance plan.

\section{BIBLIOGRAPHY}

[1] Merle E. et al Installation of the AIRIX induction accelerator Proc. Of LINAC98, Chicago, USA, August 1998.

[2] Ribes JC. et al RBF classifier for the AIRIX accelerator fault diagnosis Proc. Of LINAC2000, Monterey, USA, August 2000

[3] Demartines P., Herault J. Curvilinear component analysis : a self -organizing neural network for non linear mapping of data sets. IEEE Trans. On Neural Networks, Vol 8, pp 148-154, 1997.

[4] Ribes JC. et al Faults diagnosis on the AIRIX induction accelerator Proc. Of IAR-ICD Worshop, Nancy, France, November 2000. 